\title{
Metaphors to Survive by: Mimicry as Biometaphors, Embodiment of Sign and Cognitive Tools (not only) in Animals?
}

Original Study

Róbert Bohát

Institute of the Czech National Corpus, Faculty of Arts, Charles University in Prague

Received: January 2021 Accepted: April 2021

\begin{abstract}
Can Cognitive Metaphor Theory (CMT) be applied productively to the study of mimicry in zoosemiotics and ethology? In this theoretical comparison of selected case studies, I would like to propose that biological mimicry is a type of biosemiotic metaphor. At least two major parallels between cognitive metaphors in human cognition and mimicry among animals justify viewing the two phenomena as isomorphic. First-from the semiotic point of view-the argument is that both metaphor and mimicry are cases of semiotic transfer (etymologically: metaphor) of the identity / sign of the source onto the perceived identity / sign of the target. This identity transfer, in turn, triggers appropriate changes in the response (behavior) of the surrounding (human or animal) interpreters (e.g. predators). Semiotically, the mimicry turns the body of its bearer into a sign of something else, resulting in the interpreters' (e.g. predators') perception of species $\mathrm{X}$ as species $\mathrm{Y}$-hence, a type of embodied sign and cognitive metaphor. Second, ecologically, a species occupying one niche (e.g. a moth: non-venomous, herbivorous primary consumer) is perceived and identified as an occupant of a different niche (e.g. a hornet: venomous, omnivorous predator). Thus, a potential predator's Umwelt is affected by its perceiving a hornet moth as "a hornet" where there is, in fact, a moth, and its response to this stimulus will not be predation but avoidance. In terms of $\mathrm{CMT}$, we could call this a biosemiotic metaphor (bio-metaphor), e.g. "A MOTH IS A HORNET" or "PREY IS A PREDATOR". Further correspondences between mimicry and metaphor include the fact that this bio-metaphorical identification by mimicry does not typically require a "perfect" resemblance between the source and the target sign (or species); this seems to correspond to the prototype categorization in CMT where categories are "open-ended" and only a partial similarity is sufficient for metaphorical identification (compare Lakoff, Johnson 1980; Rosch 1983). Such an identification of mimicry as metaphor could be based on Prodi's argument that "hermeneutics is not a late product of culture, but the same elementary movement of life that is born because something obscurely interprets something else" (Eco 2018: 350; Kull 2018, 352-364). Inasmuch as animal Umwelten are interconnected inter alia by this natural hermeneutics, the transdisciplinary approach to the study eco-zoosemiotic interpretants on the basis of metaphor-mimicry isomorphism could open new opportunities in comparative studies of semiosis in human and animal cognition and interactions.
\end{abstract}

Keywords: biosemiotic metaphor (biometaphor), embodied sign, mimicry, metasemiosis, metasign, minimyth, non-verbal metaphor, rudimentary metaphoricity, semiotic continuum.

\section{INTRODUCTION}

"Go to the ant...observe its ways and become wise," said the wise King Solomon (Proverbs 6:6, author's translation). Imagine taking this encouragement to heart and going out in search of an ant to observe. Depending on which part of the world we live in, we could come upon a busy ant worker pulling a caterpillar on a tree. Somewhere else, we could see a weaver ant carrying a minor worker, or a group of black ants on a leaf, frantically foraging for food. We can learn a lot from these "creature teachers": industriousness, helping the weak and leading a proactive life. And-upon closer inspection-a most important lesson: that appearances may be misleading.

In fact, what we might have observed in the first case was a single larva of the lobster moth (Stauropus fagi), 
a part of whose body looks like an ant and the rest like a writhing caterpillar. The second "ant" might have been a Kerengga ant-like jumper, i.e. a spider (Myrmarachne [or Myrmaplata] plataleoides), while the third "ant" could have been nymphs of the Asian ant mantis (Odontomantis planiceps). Thus, due to mimicry (in this case: myrmecomorphy), "going to the ant" may be more of a challenge than it seems. Hence, we could ask: if we humans can be misled by the apparent "ants," how much more so their potential predators, who seem to lack the level of metacognitive reflection of humans?

Thus, it is only understandable and (all too) human to feel deceived by these creatures and therefore to project human ethical or cultural categories onto these animal mimics. Sebeok $(2001,19)$ summed it up fittingly: "Deceptive nonverbal signalling pervades the world of animals and men'1. In animals, basic shapes of unwitting deception are known as mimicry." Popular science literature and films as well as academic papers (prose) often label mimicry as "deception," "lies," "unmasking nature's imposters," "tricksters," "honest vs. dishonest" signaling, "cheats," etc., (Lebas, Hockham 2005; Blount et al. 2008; Forbes 2009; Gohli, Högstedt 2010; Summers et al. 2015; etc.). However, such projection of human culture onto non-human nature is problematic for several reasons; one of them being that evaluating natural phenomena with moral categories of "dishonesty" could distort our understanding of these phenomena more than is inevitable by contributing to a negative stereotype of a species and blind us, even partially, to its natural roles. A degree of anthropomorphism is unavoidable in human cognition, but given the fact that "imposters" and the like carry a strong emotional charge of condemnation, ${ }^{2}$ this could potentially and unnecessarily complicate human attitudes toward these species and their conservation.

Cognitively, these ethical categories apply meaningfully to us humans with our cognitive make-up and ability to understand and evaluate them, but not to organisms with different levels and types of cognition. Just as some animal categories cannot be transferred to humans (such as categories perceived by bat echolocation, insect pheromone communication, etc.), so there are some human categories that are non-transferable to animals (including ethical categories). Hence, it seems beneficial to consider avoiding the use of moral or ethi$\mathrm{cal}^{3}$ vocabulary in describing non-moral (or non-ethical) natural phenomena such as mimicry and replace this loaded terminology with terms that will be closer to what can be considered natural (and therefore ethically neutral). To illustrate, we could describe a venomous model's aposematism as factual signaling and the non-poisonous mimic's could be termed counter-factual signaling. ${ }^{4}$ Anthropomorphism is indeed unavoidable in human discourse, but it can be applied within reasonable limits: describing the natural in terms of nature, and the cultural in terms of culture, and not mixing the two like "apples and oranges."

This problem of the inevitable anthropomorphism raises the question of the comparability of human and animal cognitive processes and categories. Given that all organisms on Earth make up a biosemiosphere of partially overlapping Umwelten, some cognitive overlaps seem to be both possible and appropriate. The goal of this paper is to argue that one such cognitive overlap is the concept of metaphor. Contrary to popular opinion and scholarly traditions, Conceptual (Cognitive) Metaphor Theory argues that metaphor is not a late product of a cultural embellishment of language, but a primary cognitive tool of the embodied human mind (Lakoff, Johnson 1980; Lakoff 1998). If that is the case, then applying rudimentary metaphoricity to some aspects of animal cognition would be comparing what is natural in humans with what is natural in animals. Furthermore, the above examples of myrmecomorphy are more than just a passive "ant-shaped appearance"-this dynamic, event-like form of mimicry creates an unfolding sequence of actions (a "story") and what happens by means of these actions turns out to be quite different from what it appears to be: an enacted metaphorical sequence. Hence, there seems to be a commonality between human and animal semiosis not only in terms of metaphor itself (identifying one thing as an unrelated other), but also

1 Humans sometimes "embody non-verbal metaphors" when faced with human "predatory" pressures: as in the story of Istvan Faludi (Friedman) whose "shapeshifting skills ... saved his life" during the Nazi occupation in Hungary when he "rescued his parents from deportation by impersonating a fascist Arrow Cross officer" (Haldeman 2016; Hyland 2017). Similarly, Solomon Perel was a Jewish boy who survived WWII as an "Aryan" member of Hitlerjugend (Perel 1997).

2 Famously, in the Western Civilization, Psalm 26:4 says: "I have not sat with men of falsehood; neither will I go in with dissemblers [i.e., those who conceal or hide what they are]." Human society is justified in condemning such imposters as dishonest hypocrites, but animal communities cannot be coherently judged by human ethical values and standards that make up a culture in which animals do not participate meaningfully.

3 Ethics or morals are co-extensive with human culture, not with nature per se. It is also methodologically appropriate to use natural terminology in describing natural phenomena, and cultural terms to account for sociocultural phenomena.

4 Alternatively, categories of "true" and "untrue" aposematisms can be used in this way based on their value in logic without moral condemnation; a statement may be (onto)logically untrue and yet have no taint of "dishonesty" or "deception". Similarly, it seems reasonable to avoid terms like "fake" or suffixes like "-oid". 
a primary form of "narrative," a metaphorical (symbolic) story, a myth of sorts, or a mini-myth ${ }^{5}$.

These semiotic phenomena of biosemiotic metaphor and / or biosemiotic myth influence inter-specific as well as intra-specific interactions ecologically due to each species' interpretations as part of their navigation of their Umwelt. ${ }^{6}$ This art of symbolic or metaphorical representation in animals seems similar to human rituals, as Komárek says: "Classical ethology also points out another source of mimetic phenomena in nature-it is ritualization, where a complete action is replaced by representational action or displacement activity" (Komárek $1998,115)$. He illustrates this with the domestic rooster that offers some food to the hen before mating, while a pheasant male (genus Lophura) "only acts out this behavior without actually offering anything-the action here has a representational value, it is symbolic." This representational, symbolic value of a series of "empty" actions reminds us of human rituals:

Everyday personal rituals are also experiential gestalts consisting of sequences of actions structured along the natural dimensions of experience-a part-whole structure, stages, causal relationships, and means of accomplishing goals (Lakoff, Johnson 1980, 234).

They also argue that at least some rituals may be "metaphorical kinds of activities" (Ibid.). Thus, if at least some rituals are enacted metaphors (where an action stands for another action in a representative way), then it can be argued that a degree of rudimentary metaphoricity and narrativity can exist in some types of animal cognition and related semiosis.

The use of analogies in biological studies has had its critics and defenders. Critics warn against false analogies and misapplied concepts, and these warnings need to be taken seriously, as we demonstrated on the use of "deception" terms above. On the other hand, Konrad Lorenz argued in his Nobel Prize lecture that "no such thing as a false analogy exists: an analogy can be more or less detailed and hence more or less informative" (1973, 104-105). Speaking of the concepts of "friendship," "enmity," etc., among animals, he argued:

These terms refer to functionally-determined concepts, just as do the terms legs, wings, eyes and the names used for other bodily structures...in different phyla or animals. No one uses quotation marks when speaking or writing about the eyes or the legs of an insect or a crab, nor do we when discussing analogous behavior patterns...These examples are sufficient to demonstrate the importance of keeping functional, phylogenetical and physiological conceptualizations clearly apart (Lorenz 1973, 104-105).

Hence, even if we do not fully agree with his claim that "no ... false analogy exists," we can agree that "recognizing analogies can become an important source of knowledge" (Lorenz 1973, 104-105). So, when popular science describes mimicry as "the art of appearing to be something else"-a description that would fit several aspects of human behavior, including metaphor, ritual, narrativity and theatrical acting -it seems appropriate to ask whether the human and animal versions are analogous (similar) or even isomorphic (identical) (Main 2019). Furthermore, in the absence of evidence that animals possess ethical categories, we would be able to delineate the limits of appropriate analogy by avoiding ethical terms such as "imposter," "deception," etc.

In other words, the question explored here is whether mimicry is to animal cognition what metaphors are to human cognition. Biosemiotically speaking, are the bodies (or body parts) of mimicry organisms cases of embodied signs involved in a higher-level semiosis (could we call this meta-semiosis?), communicating what is not the case to an animal observer (and potential mate or predator), thus increasing the likelihood of its survival and/or reproduction success? Thus conceived, are they coherently and consistently construed as analogous or even isomorphic to cognitive metaphors in humans? Are mimicry embodied (bio)metaphors to live and survive by? And, are their enacted, sequential (event-like) versions a rudimentary form of a myth, a mini-myth?

\section{SEMIOTIC ISOMORPHISM: MIMICRY AS METAPHOR}

Exploring the possibility and / or degree of isomorphism between metaphor and mimicry could start by analyzing their definitions expressed in mathematical symbols. The thesis of this argument is that one way to generalize various definitions of metaphor semiotically is as follows: Metaphor (Me) is an identification of (sign) A as (sign) B if and only if A is not B. In mathematical symbols, this paradox of metaphor can be expressed as follows: $\mathrm{Me}=\{\exists \mathrm{A}=\mathrm{B}<=>\mathrm{A} \neq \mathrm{B}\}^{7}$. Similarly, we can have a parallel thesis (parathesis); mimicry (Mi) could be simplified as an identification (perceived equality) of an organism of species $A$ as an organism of species $B$ if and only if species $A$ is not really species $B$. Mathematically,

$M i=\{\exists A=B<=>A \neq B\}^{8}$. Hence, the synthesis: if $M e=$ $\{\exists A=B<=>A \neq B\}$ and $M i=\{\exists A=B<=>A \neq B\}$, then it

\footnotetext{
5 Compare the term "mini-myth" for metaphor and mimicry proposed here with the concept of monomyth by Campbell (2008).

6 Sometimes these semiotic "myths" (counterfactual stories) affect a species' chance of survival-compare the Australian jewel beetle (Julodimorpha bakewelli) males who consider beer bottles their females (Williams 2011).

Pheromone traps are another example of the use of biosemiosis to manipulate the ecological network of an ecosystem.

7 In English: "Metaphor (Me): There exists (is) such an A that is B if and only if $A$ is not B."

8 In English: "Mimicry (Mi): there exists (is) an organism of species A that is an organism of species $B$ if and only if it is not (in fact) organism B."
} 
follows that $\mathrm{Me}=\mathrm{Mi}$. Therefore, at this level of semiotic analysis it seems justified to consider metaphor and mimicry as procedurally isomorphic.

Can this proposed isomorphism of metaphor and mimicry be exemplified in a case study? Let us compare a famous metaphor identifying two different species with a case of insect mimicry. Using the rigorous MIPVU method of metaphor identification, a metaphor occurs when a word (or an object, person, or concept) is identified as something ontologically different from its basic meaning (MIPVU, Pragglejazz 2007). Thus, as demonstrated above, metaphor is a cognitive paradox of identification of something as what it obviously is not. Within this framework, the famous words of John the Baptist: "Ecce, Agnus Dei" ("Behold, the Lamb of God") 9 could be literal or metaphorical depending on the reference point; if, in fact, he had pointed to a young sheep in the Temple in Jerusalem, this would have been a literal description because the basic meaning of "lamb" is identical with the object so described, a young specimen of Ovis aries. Since, however, John's reference was to Jesus of Nazareth, belonging to a different species (Homo sapiens), this is a metaphor indicating the human "lamb's" goal or function, including his mildness and willingness to make a sacrifice for others. If $\mathrm{H}$ stands for a "human" and $L$ for "lamb", then: $\exists H=L<=>H \neq L$, which corresponds to the definitions of metaphor and mimicry above. Similar cross-species metaphors are abundant and well documented in human semiosis, as in cognition and communication.

The case of one of Western civilization's key metaphors across two biological species can be used as a parallel to the hornet moth's (Sesia apiformis) mimicry. Due to its (not only) morphological features, humans and animals alike typically perceive this moth (from the order of Lepidoptera) as a hornet (Vespa crabro, from the order Hymenoptera). ${ }^{10}$ This identification of a harmless moth as a harmful hornet is a case of perceiving one species as another with the appropriate avoidance reaction among humans and animals and is defined as mimicry. If, in fact, the insect so identified turns out to be a hornet, it would not be a case of mimicry, but perhaps polymorphism within the species. In mathematical symbols, if $\mathrm{M}$ stands for the hornet moth and $\mathrm{H}$ stands for the hornet, then: $\exists \mathrm{M}=\mathrm{H}<=>\mathrm{M} \neq \mathrm{H}$ (which matches the above definition of a metaphor). In terms of mimicry terminology, the mimic (hornet moth) is perceived as identical with the model (hornet) by the signal-receiver (or "operator," such as a potential predator or a human observer, cf. Maran 2007). Thus, the Lepidopteran moth is identified as what it is not (a Hymenopteran hornet)and thus benefits by decreasing the likelihood of becoming a potential predator's prey. It is "a sheep in a wolves' clothing," indeed, a metaphor in the Animal Kingdom, a metaphor to survive by.

Thus, the above case studies of cross-species metaphor and mimicry seem to support the isomorphism of mimicry and metaphor. Hence, if it indeed is the case that metaphor and mimicry are isomorphic, and the predator actually perceives (recognizes) the moth as the hornet, then the moth has become an embodied sign of a hornet, and this embodied sign (of a hornet which is not there) is processed cognitively ${ }^{11}$ by the signal-receiver (typically an animal predator without human language). Hence, this could be a case of embodied cognition in animals, and an interesting confirmation of the metaphoricity as a matter of cognition rather than a "superficial" matter of language (cf. Lakoff, Johnson 1980). In other words, a metaphor seems to be brought out in this case as a cognitive identification of non-identical objects, concepts or organisms, i.e. (in terms of Lakoff, Johnson's terminology), a cross-domain mapping of $A$ as $B, X$ as $Y$ (provided $A \neq B$ and $X \neq Y$, and $A \notin B ; X \notin Y$ ). This cognitive identification by metaphor is paradoxical. It is "true" on condition that the physical and literal identification of the two is not true (otherwise it would be a straightforward identification or categorization); this way, the metaphor's truth depends on its "untruth," so to speak. The same seems to be happening in mimicry. Therefore, mimicry would be a special case of cognitive metaphor, and Cognitive Metaphor Theory (CMT) might be of use in biology in general, and biosemiotics specifically. The only cognitive difference seems to be that while a human observer (qua signal-receiver) may be(come) aware of the non-literal meaning of metaphor or the fact that the hornet moth is in fact not a hornet, animal signal-receivers seem to be generally lacking in this capacity to "see through" the mimicry (as far as we can tell from the consistency of avoidance or attraction triggered by mimicry). This could be due to our human capacity for metacognition-thinking about our thinking.

\section{SIGN, METASIGN, AND EMBODIED (META)SIGNS}

Similarly, a comparison of the semiotic (Peircian) sign system for metaphor and for a case of aposematic mimicry shows that they are both metasemiotic processes involving a sign, and a sign of a sign (metasign),

9 These words from the Gospel of John (chapter 1, verse 29) in Latin (or other languages) can be seen on buildings, works of art, and heard in music all over the Western world, as well as in Eastern Christianity. 10 Although its scientific name "apiformis" points to the fact that the taxon's author (Clerck 1759) thought it similar to Apis (the honeybee), many (though not all) modern languages seem to see it as similar to the hornet (English: hornet moth, Czech: nesytka sršňová, Slovak: podobník sršňovitý, etc.).

11 This cognitive processing means that the sign is decoded and interpreted, to use more common zoosemiotic terms. Here, the expression "processed cognitively" is intended to highlight the cognitive nature of these processes, leaving the question about the potential distinction between the terms for another discussion. 
i.e., these two phenomena are semiotically isomorphic. In the semiosis of metaphor, the process is at least twofold. Primary semiosis occurs when an object (e.g. a lion-species $A$ ) is represented by a sign (e.g. a word "lion" or a picture, etc.). In human cognition, the sign is one of the key cognitive tools of human interpreters' "slicing up the world" into categories and concepts for better and safer orientation, and navigation within it (see Figure 1 below). Once this sign becomes meaningfully known to the interpreter, the same sign can then be applied to another object normally represented by a different, unrelated sign-such as the word "human." Hence, we represent one sign "human" (perhaps even a specific name) by another (unrelated) sign "lion" (such as when Jesus of Nazareth is called "The Lion of the Tribe of Judah").12 Thus, at the beginning, at least two signs are identified directly, in the primary semiotic categorization of our Umwelt. Then on the second level, we use one sign to describe another sign, making it a sign of a sign (a metasign). For this reason, it seems appropriate to describe this second level of semiosis as metasemiotic.

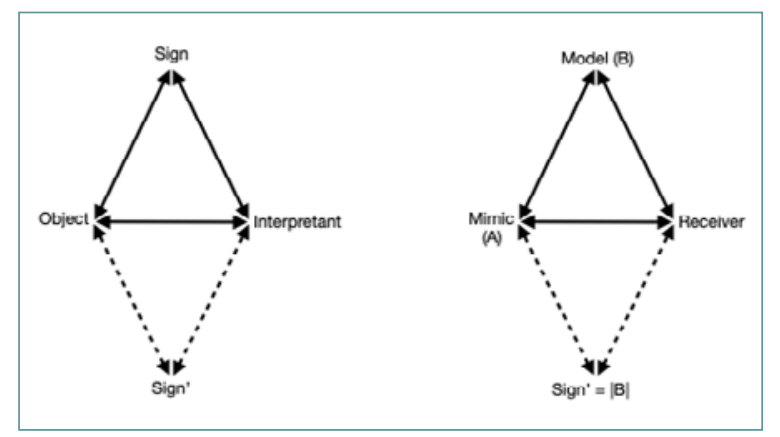

Figure 1: A comparison of a metaphor sign system (left) and aposematic mimicry sign system (right). The Object-Interpretant and Mimic-Receiver lines separate the direct semiosis (above the horizontal line) where a sign is a factual representation of an object (left) and a model organism's aposematism is a factual warning coloring. Below the horizontal lines are diagrams of metasemiotic phenomena; Sign' is a metaphorical sign representing the object in a non-literal way; similarly, in the mimicry system, a harmless mimic's imitation of a noxious aposematic model is Sign' identified (metaphorically) as the Sign of Model B.
Similarly, the process of mimicry semiosis seems to be twofold. First, there is the categorization in straightforward primary semiosis ("it is what it is" type); a hornet's (Vespa crabro) body with the aposematic yellow-black coloring and typical movements and sounds becomes an embodied sign meaning "danger," with the animal predator interpreter's memory storing a previous negative experience, triggering the fight or, more often, flight reaction. The same color pattern in a hornet moth (Sesia apiformis) is perceived by potential predators as "identical" with the original object from their experience (the harmful, noxious hornet) and will typically also trigger the fight or flight reaction (avoidance rather than predation). Hence, the sign of the hornet is embodied in the phenotype of the moth (which is not a hornet with a sting but appears to be one). The hornet moth's body is interpreted as a harmful hornet in the potential predator's Umwelt ("it is what it isn't" type of interpretation: see Figure 2 below). Thus, it appears that the two-step shift from semiosis to metasemiosis happens here, too. Interestingly, signs in animal mimicry are embodied-they may include the whole body or body parts and aspects (such as coloring, movement, body positioning and movement, sounds, scents, etc.). As exemplified by the hornet mimicry case study, animals sometimes have real reactions to unreal actions (like a cuckoo avoiding a hornet moth rather than eating it-the avoidance is a real reaction, but the hornet is not really there). Similarly, humans shed real tears of joy or sorrow when watching fictitious "events" (films, theater) unfold, even though they are fully aware of the fact that the "events" are unreal-that they never really happened. This similarity illustrates another aspect of mimicry as something "fictional" and "figurative" in animal cognition, very much like in human cognition (just to a lesser degree).

Given the fact that some forms of mimicry involve movement and others do not, we could analyze these not only as biometaphors representing one animal as another, but also discern a degree of "eventfulness" or "narrativity" in some mimicry phenomena. Some types of mimicry have the interchange, substitution, or identification of two physically unrelated objects or organisms in terms of each other. For example, "terrestrial orchids of the genus Ophrys, with their uncanny flowers shaped like insects...attracting male bees with an imitation of sexual pheromones" (Komárek 2009, 171). At this level, the plant to be pollinated looks and smells relatively "statically" like a female bee ready to mate-this triggers

12 In the book of Revelation 5:5. Interestingly, the foundation text of the Western Civilization uses two different species of animals to describe the same human metaphorically-thanks to metaphor, a human can be at once a "lamb" as well as a "lion"-counterintuitively combining mildness with courage. Unlike ethical categories of "dishonest imposters," etc., mentioned above, these metaphorical uses of "lamb" and "lion" reflect observed behavioral patterns in both animals (namely, calm meekness versus fearlessness) and project their qualities onto humans in what is explicitly understood as metaphors. Hence, this is not a case of a "cultural category trap" criticized here, as animals are not described in human ethical terms, but humans are conceptualized metaphorically in animal terms. Furthermore, it demonstrates the creative and inventive power of the (not only inter-species) metaphor to go beyond the customary and habitual, to be the engine of human progress. 


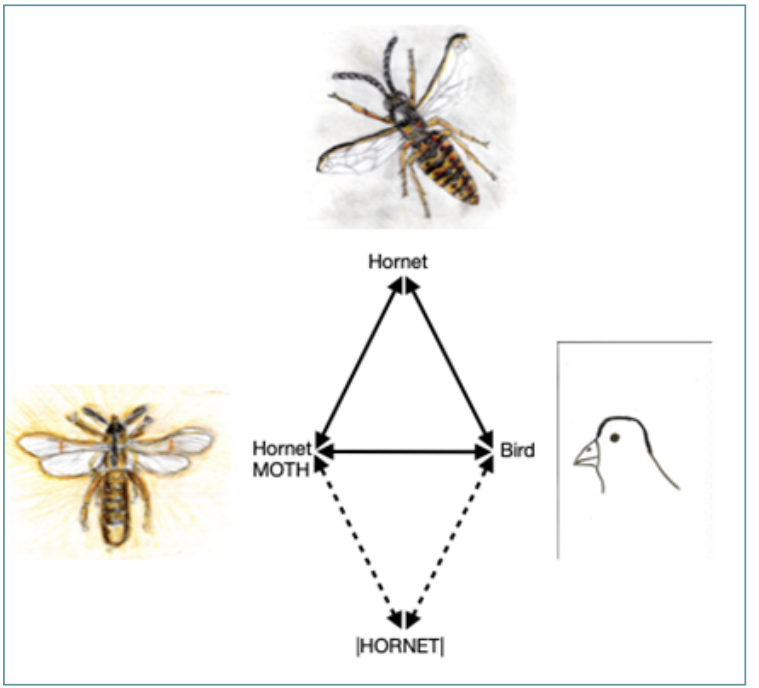

Figure 2: A semiotic representation of hornet mimicry (a biosemiotic metaphor or "biometaphor"). The hornet moth (Sesia apiformis) becomes an embodied sign of the hornet's (Vespa crabro) aposematic sign of warning. (Drawings: hornet by Jan G. Martinek; hornet moth by Jakob G. Martinek; bird by Eliáš R. Bohát)

the mating behavior reaction in the males. Yet, other forms of mimicry involve apparent action, such as the hornet-like body movement and buzzing in the hornet moth, the apparent struggle of the nonexistent "ant" with the "caterpillar" in lobster moth larvae or the illusion of ant-to-ant helpfulness in the jumping spider. Similarly the spider-tailed horned viper (Pseudocerastes urarachnoides) of Iran enacts a sequence of movements of its tail tip shaped like a spider as if a careless spider was crawling on a rock (caudal luring-compare Bostanchi et al. 2006; Fathinia, Rastegar-Pouyani 2010; Fathinia et al. 2015). This attracts the spider's bird predator who flies in to catch the spider (i.e., to become its predator). Thus, this type of mimicry leads to the predation based on predation and the would-be predator becomes prey in the process (see Figure 3 below). Yet, it is the untrue, counter-factual nature of the embodied sign of the viper's tail that tells the "tale" of a spider where there is in fact a snake's tail. The "SNAKE TAIL IS A SPIDER" seems to be the metaphorical identification.

Such more complex, event-like mimicry (mimetic action) seem very similar to human narrative fiction-a series of events that seem to be happening but in fact are not. It is sequential and "fictional" at the same time. In human cognition, relating a sequence of events that did not really happen is called a tale, a story, a myth..$^{13}$ These types of mimicry seem to be doing the same thing at a rudimentary level of animal cognition: en-acting (as in the theater) a sequence of actions or events that represent

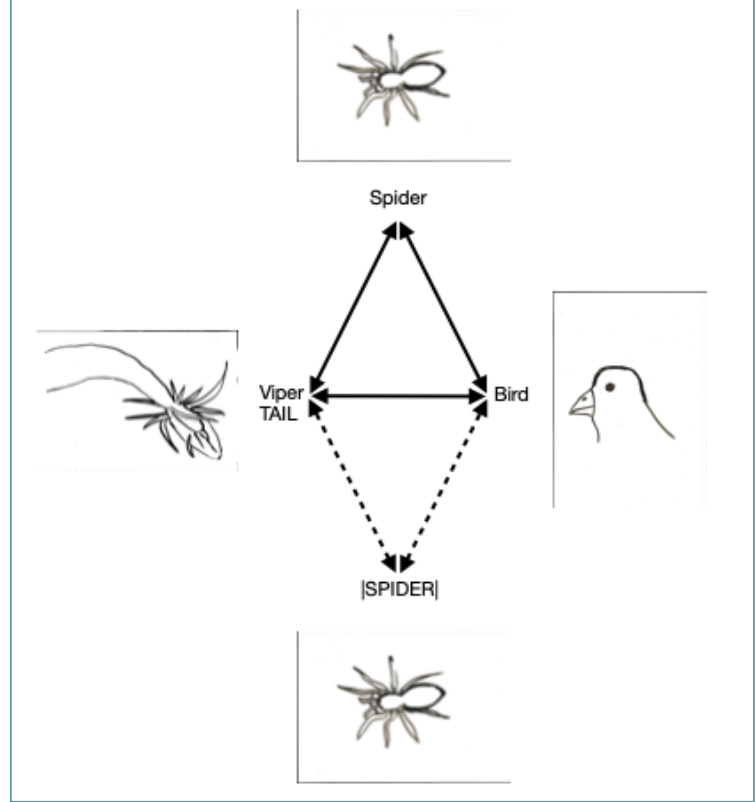

Figure 3: Event-like mimicry in caudal luring by the spider-tailed horned viper (Pseudocerastes urarachnoides); the viper's tail not only looks like a spider, but also moves like one to attract a spider predator (a bird, a lizard, etc.) who will become the viper's prey. The biometaphor: "SNAKE TAIL IS A SPIDER" seems to have a sequential, "narrative," myth-like nature. (Drawings: viper's tail and bird: Eliáš R. Bohát)

(stand for) a set of different actions. Would it, then, be appropriate to see this isomorphism as a sufficient justification for calling event-like mimicry a primary level of narrative fiction, a primary myth? If so, then myth-making would not the exclusive privilege of humans, but shared-to a degree-with our animal fellow terrestrials.

These examples and analyses of mimicry seem to justify the interpretation of mimicry as a primary, biological form of cognitive biosemiotic metaphor ("biometaphor") and dynamic mimicry that seem to be "spinning" story-like sequences as a primary level of narrative semiosis, a metaphorical story such as a parable or a myth (hence, my proposal here to call it a "mini-myth"). Semiotically, these phenomena seem to be isomorphic in terms of their basic definitions and procedures. They also share the power to trigger similar effects on other organisms' behavior-either a fight or flight reaction (or attraction, predation or avoidance)-just like their human counterparts of metaphor and myth can trigger strong emotional reactions. Therefore, if these analogies prove to be justified, they could help us get a deeper and more authentic glimpse into animal cognition and categorization based on metaphorical processes and categories and take another step towards understanding what it is like to be a bat (or a cat, or a gnat-compare to Nagel 1974).

13 Obviously, some stories or tales ("myths") describe events that did happen while others may express truths and values in a counterfactual manner (independent of their historicity-e.g. metaphors, parables); still others are outright lies. Technically, the term "myth" can entail the whole spectrum of meanings. 


\section{BIOSEMIOTIC AND ETHOLOGICAL IMPLICATIONS: THE DUCK-TEST OF A SIGN}

Analyzing mimicry as a type of cognitively metaphorical process may lead to a productive application of another aspect of CMT in a deeper analysis of animal cognition and behavior, namely prototypes. In human cognition, much of metaphorical productivity is possible due to the real or projected similarity between the prototype of the source domain and a prototype from the target domain (Rosch et al. 1978; Rosch 2013; Lakoff, Johnson 1980). Thus, when a human poet says, "I wish I had wings," they typically do not refer to ostrich or penguin wings (which are real wings but not prototypical as they do not enable flying), but the prototypical wings, such as those of a dove or an eagle. The eagle's wings are closer to the prototype of a wing because they make it possible for the eagle to fly (and then even technological terminology can use this similarity in calling the lateral extensions of the airplane "wings"). These artificial airplane "wings" are "good enough" in approximating the prototype to the point of creating the impression of similarity and ease of identification and categorization.

Similarly, in animal cognition and categorization it has been reported that the mimic does not always produce a very accurate icon of the model; the "imperfection" of the mimicry is often emphasized as puzzling in view of its efficiency (e.g. Harper, Pfennig 2007; Speed, Ruxton 2010). Cognitive prototypes could help us understand this seeming paradox: how can a rather "imperfect" case of mimicry fool a potential predator so successfully? Prototypes of categories have been described as "the clearest cases of category membership defined operationally," involving "perception of typicality differences" (Rosch 2013). If animals navigate their Umwelten on the basis of perceived prototypes, then it may be that the aposematic coloring of the harmless hornet moth is not cognitively assessed on the basis of how "closely" it resembles the hornet, but on the basis of its iconic proximity to a more generalized prototype pattern of aposematic coloring-in this case, the iteration of yellow and black or bright and dark color stripes ${ }^{14}$ (Schuler, Hesse 1985; Skelhorn, Rowe 2010; Stevens, Ruxton 2012;
Arenas et al. 2014). If the prototypical sign is something like "yellow/black iteration" spells "danger," then one encounter with a wasp or hornet could be enough to activate this sign identification with all its "variations." Thus, one of the prototypical signs in zoosemiosis could be the iteration of yellow and black (and their shades) or even the repeated pattern of bright-and-dark..$^{15}$

Prototypicality could thus be at work in the biosemiosis of mimicry. As we have seen, there are various types of bird wings-some closer to the prototype than others-yet, having a certain minimum of characteristic features helps us identify an ostrich wing as a bird wing despite the absence or weakness of some other features (like the ability to fly). Similarly, the coloring patterns similar to the prototypical "danger sign" (in hornets and wasps) could serve animals as a relatively quick and simple guide for a fast and safe navigation of the ecosystem (and their Umwelt). And since there seems to be a whole range of variations of this aposematic pattern, it may be that there is a prototype-based, biosemiotic continuum of signs involved in mimicry. In the case of the "yellow-jacket prototype," we can discern a continuum from the genuine hornets, wasps and bees on one end through to the hover flies, wasp mantidflies (mantispids), hornet moths, wasp spiders, and wasp beetles. Some of the mimics are visually or behaviorally closer to the prototype than others, and they have different degrees of semiotic success in predation avoidance-but all of them seem to benefit from it. This continuum includes factual aposematic warning (of truly noxious or venomous species, i.e., hornets, wasps, and bees) as well as the counter-factual aposematism that sends the warning but is not in fact dangerous (the "sheep in wolves' clothing," to paraphrase the Biblical metaphor). ${ }^{16}$ Thus, identifying hornets and wasps as "yellow-black danger" would be a direct, straightforward semiosis (from sign to accurate/true interpretation of "it is what it is") while identifying hover flies or hornet moths as "danger" would be a case of "it is what it isn't" semiosis, in other words, a biosemiotic metaphor.

If the prototype-based semiosis and cognition work in animals, this could be a basic "Duck Test" of animal

14 The increased visibility of the bright colors makes the bearer of the aposematic sign unmissably visible, therefore, more likely to attract a new predator's attention early on in the predator's life. This experience will then be remembered as a basis for the quick recognition of this bright, visible coloring as a potential source of danger to be avoided (cf. Schuler, Hesse 1985).

15 We, humans, sometimes generalize similar prototype patterns in memory aids, such as the coral snake rhyme "Red on Yellow, Kills a Fellow; Red on Black, Venom Lack" (with many variations). This North American traditional lore is meant to provide a quick distinction between the venomous coral snakes (from the family Elapidae) and their typically harmless mimics king snakes and milk snakes (from the family Colubridae). Herpetologists are aware of the fact that polymorphism and/or color aberrations and anomalies among the different species account for exceptions to this rule; yet-as a general rule of thumb, the rhymed "prototypes" are reported to be helpful for quick orientation (e.g. Simbeck 2008).

16 Compare with what humans often do, as Proverbs 13:7 puts it fittingly: "One person pretends to be rich, yet has nothing; another pretends to be poor, yet has great wealth" (New International Version). Similarly, some animals appear to be dangerous, yet have nothing to hurt with; others "pretend" to be harmless, yet have dangerous poison or venom. 
semiosis; if it looks like a prototype, moves like a prototype, sounds like a prototype and smells like a prototype, then it "is" that prototype. The mimic's body 'becomes a sign' of another animal type (compare with Maran 2011). Thus, zoosemiosis involves, inter alia, bodies, body parts, functions, and secretions as signs (embodied signs when identifying the prototype factually, i.e. when it really is the animal type signified) or metasigns (i.e., biometaphors when identifying figuratively or counter-factually, when it really is not the animal type signified). Just as in human cognition, prototypes (and/or stereotypes, archetypes) simplify and economize the cognitive processes involving semiosis by speeding up categorization, identification of food, mates, or danger and allow more time and energy for carrying out the appropriate action rather than spending them on a more finessed recognition. This semiotic simplification (like all simplifications) comes at a cost (less accuracy in identification, or "lower resolution" in identification, higher frequency of "false positives," as in mimicry, etc.), but the historical success of the known mimics as well as their models seems to indicate that the overall advantages outweigh the disadvantages.

\section{ECOSEMIOTIC ${ }^{17}$ IMPLICATIONS OF A BIOMETAPHORICAL SIGN}

Applying CMT to our conceptualization of mimicry could lead to an application of its way of describing metaphors to the description of mimicry. CMT uses capital letters to describe the identification of the target and source domains, such as in the famous "LOVE IS A JOURNEY" metaphor (Lakoff, Johnson 1980). Similarly, taking the hornet moth (Sesia apiformis) mimicry, we could describe the biometaphor as "A MOTH IS A HORNET," and in parallel, other members of the 'yellow-jacket' semiotic continuum could be described as "A HOVERFLY IS A WASP; A SPIDER IS A WASP; A BEETLE IS A WASP," etc. Such representation is potentially useful in analyzing interspecific interactions in an ecosystem made of several overlapping Umwelten. The following paragraphs present a few examples of biometaphor impact on inter-specific trophic relations, such as predator-prey, parasite-host, etc. These impacts have implications in terms of the overall ecosystem energy flow, the reproductive efficiency of the species involved, as well as redefining the mimics' and the receivers' ecological niches.

Hornet moth mimicry can illustrate the first example of biometaphor impact on trophic relationships. The common cuckoo (Cuculus canorus) normally hunts moths; a hornet moth is a moth, so there would be a natural predator-prey relationship between the cuckoo and the hornet moth. However, where a keen (and trained) human eye sees a hornet moth, the cuckoo seems to see a hornet and its reaction is not one of predation but avoidance. From the ecological standpoint, there is an edible biomass of moths available in a local hornet moth population, but it will remain mostly unused since in the cuckoo's Umwelt the "MOTHS ARE HORNETS." This, in turn, will lead to a more intense predation pressure by the cuckoo on other insect species, some of them potential food or niche competitors to the hornet moth. This can lead to an increase in the hornet moth population coupled with a decrease in other insect populations. A higher proportion of hornet moths in the community will then affect the trees in which their larvae develop. In this way, a biometaphor (i.e., a non-literal interpretation of the moth as hornet in the cuckoo's mind / Umwelt) can have a very physical impact on the cuckoo's behavior, the trophic interactions within the ecosystem, its dynamics, energy flow, the tree population dynamics, plant succession, etc. However, such a metaphorical identification of the moth as a hornet will not appear intraspecifically; to a hornet moth, a hornet moth is a hornet moth, nothing else. In other words, a male hornet moth will perceive another male hornet moth not as a hornet, but as a moth, and a potential mating rival; alternatively, it will perceive a female hornet moth as a potential mate, with the resulting attraction towards her. Thus, biosemiotically, the protective metaphor works towards other species, but for the 'insider' members of the same species it does not constitute a metaphor or mimicry.

Ant mimicry (myrmecomorphy) provides another example of the inter-species impact of biometaphors and just like human metaphors, biometaphors can bring their bearers advantages as well as disadvantages. "About 10,000 arthropods live as ants' social parasites" (Sala et al. 2014). This is bound to have a significant impact on a biospheric level, as the ant biomass worldwide is estimated to be some thirty times larger than that of humans (Pilátová 2014). As for the ant parasites, "many of them can intercept and manipulate their host communication systems," i.e., can influence them zoosemiotically (Sala et al. 2014). Butterflies of the Maculinea genus spend a significant proportion of their life cycle in the nests of Myrmica ants. The first three instars of their larvae feed on plants, but the fourth instar falls to the ground and using chemical mimicry imitating ant larvae, the caterpillar gets taken by worker ants into the nest. At this stage, the biometaphor "A CATERPILLAR IS AN ANT LARVA" is at work-and it impacts the source as well as the target domain species.

Once in the colony, caterpillars of Maculinea 'predatory species' directly feed on the ant larvae, while those

17 Although the prefix "eco-" in "ecosemiotics" emphasizes the role of the environment in semiotic interactions, the field of ecosemiotics does include animal to animal relations as one of the "key principles of ecosemiotics", since "most inter-species and intra-species relations-of which all ecological communities are composed-are based on sign relations" (Maran, Kull 2014, 43; compare with Maran 2020). This section focuses on such ecological aspects of intra-species and inter-species semiosis. 
of 'cuckoo species' are fed primarily by attendance workers, by trophallaxis. ${ }^{18}$ It has been shown that Maculinea cuckoo larvae are able to reach a higher social status within the colony's hierarchy by mimicking the acoustic signals of their host queen ants (Sala et al. 2014).

Interestingly, in addition to the olfactory mimicry, the caterpillars use auditory mimicry to sound like the ant queen (or ant queen larvae) and therefore get preferential treatment when the nest is in danger (Pilátová 2014; Thomas et al. 2013).

However, in some cases this queen mimicry may backfire; the mortality of Maculinea arion caterpillars was reported as:

nearly 3 times higher in nests that had queen ants present. This is attributed to 'queen effect,' which causes worker ants in nests with queens to attack large ant larvae (gynes) that would otherwise develop into new queens (Thomas, Wardlaw 1990).

So, when caterpillars resemble the future queen larvae while there is an active queen present in the nest, many of them get attacked and killed, and "this has been shown in previous work to be the key factor that determines changes in their abundance from year to year" (Thomas, Wardlaw 1990). Semiotically, the metaphor "A CATERPILLAR IS A QUEEN LARVA" may bring higher social status with better feeding and preferential protective treatment in one social context, but may also be interpreted as a threat to the ruling queen in a different social context. Here it seems that we are observing a context-dependent zoosemiotic interpretation of semiochemicals either as desirable or dangerous, with opposite effects to the bearer of the biometaphorical signs. And again, these biometaphors (of ant queen larvae that are not) has significant material consequences in terms of the feeding burden for the ant colony or in terms of a decrease in the $M$. arion population, depending on the context.

The biometaphors of mimicry also impact their participants' (the mimics' as well as the receivers') ecological roles and niches. For example, Lampsilis mussels have sacs of their glochidia (larvae) shaped as small fish or fish larvae. When a bass fish attempts predation, the conglutinate sacs (also known as mantel flaps) burst and release the glochidia that attach themselves to the gills of the would-be predator. Using a biometaphor "MUSSEL FLAPS ARE FISH," the mussels turn predators into parasite hosts (Barnhart et al. 2008). Thus, the metaphor "A MUSSEL IS A FISH" leads to an ecological role shift; by performing the role of a predator, the fish become parasite hosts (victims). By trying to increase their energy intake they have a long term energy loss-all because of a metaphorical sign of a fish where there was a mussel. Thus, a biometaphor produces an ecological role shi$\mathrm{ft}-\mathrm{a}$ situational paradox where "THE ATTACKER IS THE VICTIM ${ }^{19}$," and "THE PREDATOR IS THE HOST." Another way to analyze this biosemiotic metaphor is that "PARASITE IS FOOD" (as in the case of Amber Snail whose eye is invaded by larvae of Leucochloridium paradoxum (truly a paradoxical organism) which makes the eye look like a pulsating caterpillar: "AN EYE IS A MAGGOT" to a predator bird such as a finch, a sparrow, etc.).

In some cases, the mimicry metaphor involves a swapping of the predator-prey identities. "Metalmark Moths Mimic their Jumping Spider Predators" (Rota. Wagner 2006). This is a case of "THE PREY IS THE PREDATOR," a biometaphor that protects the moths by making them look like the object of their fear, as the spiders are less likely to attack members of their own species. "THE PREDATOR IS FOOD" (or "THE PREDATOR IS THE PREY") is the metaphor embodied by the Iranian spider tailed viper (Pseudocerastes urarachnoides) whose tail ending looks and moves like a spider, attracting bird predators who, in turn, become the viper's prey (Bostanchi et al. 2006). Thus, not only does the actual predator mimic prey with its tail (caudal lure, including movement, re-enacting a miniature event that is not what it seems to be) but by attacking the bird attacker it turns the other predator into its prey. The viper is a predator whose tail looks like prey (but it is not), while the bird acts like a predator and becomes prey in the process.

The success of predation (or its avoidance) sometimes depends on invisibility or irrelevance strategies and these include mimicry as metaphors to survive by in the process of "I am not here" messaging. It may take the form of "A PREDATOR IS A FLOWER" (mantis or spider species that 'merge' with the colors and shapes of their host flower petals), which seems to be an "absence metaphor," or "zero sign," as if saying: "There's nothing to see here" or "There's nothing to worry about on this flower." Any movement by these predators is likely to be interpreted as "just another flower petal moving in the wind." Here, what is not the case (the absence of a predator, therefore safety) seems to be the case. The unseen predator is more successful than the one that is easily detected.

Another example of this type of ecosemiotic metaphor is "THE PREY IS A THORN;" some treehopper species avoid predation (or at least make it less likely) by looking just like a thorn. "THE PREY IS A WASTE PRODUCT" metaphor involves treehoppers or caterpillars looking like bird droppings (including their body positioning); this, in turn makes them seem irrelevant to any visual insect predators. Various strategies of crypsis provide the benefits of "invisibility." Masked Hunter Assassin Bug nymphs (Reduvius personatus) appear like walking dust or debris, making their potential prey unable to recognize them as the threat they are; these act out the metaphor "THE PREDATOR IS DUST." Sometimes "A SEA HORSE IS CORAL"-a biometaphor whereby the

18 "Trophallaxis is the exchange of food between two individuals. The food exchanged may be salivary secretions or regurgitated gut contents" (Walker 2009).

19 The similarity of this situation to situational irony seems irresistible. 
edible seems to be inedible, the accessible seems inaccessible, the visible seems invisible-makes potential predators stay hungry even though their food is right in front of them. This seems like a natural version of the Torture of Tantalus

Preventing predation can involve the appearance of being dead or sick. The basic biometaphor here would be "HEALTHY IS SICK." This is the case in treehoppers of the genus Bocydium have orbs over their head that make them appear infected by a deadly fungus of the genus Cordyceps. Consuming them would mean risking a deadly infection. In some bird species, brood protection involves the parents pretending to be injured; in this "HEALTHY IS INJURED" metaphor, the parent plover acts like an injured bird and therefore an easy prey to distract a predator away from its nest and young ones. As soon as the predator is successfully distracted, the wing that appeared to have been broken takes the bird safely away. Thanatosis, or tonic immobility, enables living animals to appear dead (and thus of no interest to a potential predator), acting out the metaphor "THE LIVING IS DEAD" (as in the Virginia opossum "playing possum," or some species of snakes of the genus Natrix, etc.)

One of the most "poetic" form of mimicry is dummy attraction. One level of this type occurs in the fly orchid (Ophrys insectifera) whose flower shape, coloring, and scent mimic insect females, thus attracting males who by trying to mate with the dummy of a female actually perform a different form of fertilization: pollination. The orchid embodies the "FLOWER IS A FEMALE INSECT" metaphor. Yet, there is another level of creative mimicry among some orb-weaver spider species who build dummy copies of themselves (self-mimicry) on their webs, sculpting them out of debris and insect body parts. This seems to be a truly "poetic," creative activity; such act of self-mimicry-"A DUMMY IS A SPIDER" metaphor-is also similar to personification, attributing "spiderness" to non-living aggregates. These detritus decoy decorations can be understood as follows:

Sometimes an animal even alters its surroundings to fit its own image by fabricating a number of dummy copies of itself to misdirect predators away from its body, the live model, to one of several replicas it constructs for that very purpose. And this is only one among a number of iconic antipredation devices contrived by different species of a highly interesting genus of spiders known as orb-weavers (Sebeok 2001, 106, emphasis added)

Further research into Cyclosa mulmeinensis shows that webs with more decorations suffered increased numbers of wasp attacks. However because these decorations resembled spiders in size and color, they functioned as decoys to distract predators and became the focus of predator attacks (Tseng, Tso 2009).

Mathematically, the more dummies a spider makes, the lower the probability of the real arachnid being the prey.

\section{CONCLUSION}

Konrad Lorenz argued that there is no need of a magical "King Solomon's Ring" in order to learn to understand the semiosis of animals; observing their "signal code" is sufficient, just as the original Biblical story also says ${ }^{20}$ (Lorenz 2002). We may not know all there is to know about what it is like to be a bat (or a cat, a rat, or a gnat), but we may learn to decipher some zoosemiotic phenomena at least to a degree. Lorenz suggested that human-animal analogy "can become an important source of knowledge" (Lorenz 1973). This analysis presents a proposal that such analogy includes a rudimentary level metaphoricity, narrativity, and myth-making; in other words, that animal mimicry can be considered semiotically isomorphic with metaphors and thus constitute a biosemiotic metaphor or biometaphor. Just like the source and target domains merge in the minds of humans using metaphors, the signs embodied by mimics trigger model-related reactions in animal signal-receivers. And, inasmuch as these biometaphors bring their bearers survival benefits, they seem to be metaphors to survive by.

Such an identification of mimicry as a biological type of metaphor can be harmonized with Prodi's argument that "hermeneutics is not a late product of culture, but the same elementary movement of life that is born because something obscurely interprets something else" (in: Eco 2018: 350; Kull 2018, 352-364). Animal Umwelten are interconnected inter alia by this natural hermeneutics by which animals interpret mimicry as biometaphors and rudimentary mini-myths, use prototypes in (re)cognition and work with embodied signs. All of this appears to be compatible with the methods and concepts of the CMT school of thought. Hence, we can be hopeful that a transdisciplinary approach to the study eco-zoosemiotic interpretants on the basis of metaphor-mimicry isomorphism can open new opportunities in comparative approaches to human and animal semiosis, cognition and interactions. If so, there are many further questions to be asked and answered: to what extent and degree are these two types of metaphors (the human cognitive metaphors and mimicry biometaphors) analogous or isomorphic? And what are the differences between them? How can the proposed transdisciplinary use of CMT, Cognitive Linguistics and Biosemiotics contribute to zoosemiotics and ethology?

The identification of a mimicry as a type of metaphor had been previously unknown to me and emerged directly from my research into CMT and animal behavior. However, when the core of this paper was formulated, I found a paper by Coletta (from 1993) that argues a similar point: that various types of crypsis (a toad looking like a stone, etc.) and other natural phenomena are metaphorical in nature and that they constitute "figures of sight" (in parallel to human "figures of speech"). Coletta also presents an interesting concept of "metaindex" (somewhat similar

20 Lorenz refers to the original text of 1 Kings 4:33 where the Bible says that Solomon spoke "about the animals" (based on keen observation, as encouraged by Proverbs 6:6), not "to the animals" as later legends claimed. 
to my use of "metasign" above) as "a highly condensed image of biological transformation which achieves metaphoric status because it 'says' or 'sees' one thing as another... as all metaphors do." (Coletta 1993, 225) Regrettably, time did not allow a deeper comparison of Colletta's proposals and terms with mine-this would require a separate study-it is, however, appropriate and necessary to recognize Coletta's original contribution years before my analysis of metaphoricity in nature (and I do so with deep appreciation).

Understanding mimicry as a type of metaphor can open the doors to a further use of analogy and other aspects of Cognitive Metaphor Theory (including the use of prototypes, narrativity and rudimentary myth-making) in studying zoosemiosis. If that is the case, then maybe "man" is not the only "myth-maker" (cf. Frye, Jenkins 1981) Furthermore, such an application of metaphoricity can be useful in analyzing the animals' Umwelten, their niches and various aspects of their ecological relationships.

The concept of biometaphor also helps resolve the issue of moral anthropomorphism-the projection of ethical concepts onto animal cognition and behavior. When people use metaphors, we typically do not accuse them of lying, deceiving or being dishonest. When Czechs say that 'Prague is the mother of cities,' they are not accused of lying because Prague is not a female human with children who become cities. The metaphor is assessed on the basis of its poetic or cognitive value, not according to the ontological truth value. Similarly, conceptualizing mimicry as a form of cognitive biometaphor can help replace the morally judgmental terminology with a morally neutral and scientifically coherent one. Furthermore, it can help us explore deeper parallels and analogies between human and animal cognition, moving us at least one step closer to an understanding of 'what it is like to be a bat.'

Eliminating "dishonesty" or "deception" from the concept of mimicry does not mean that no advantage or disadvantage is ever incurred by any organism involved. Just like human metaphors can be beneficial, neutral or harmful, so also a biometaphor may have positive, neutral or negative impacts on the organisms involved. Yet, unlike "lies, deception and dishonesty," 21 the concepts of "benefit and harm" can be directly observed in nature.

So, heeding King Solomon's advice to "go to the ant" (as well as to its mimics) can help us learn the art of keen observation and remind us of the important fact that appearances can be misleading. And more than that: it can also help us refine and develop our biosemiotic concepts and terminology-to make them a little closer to the real nature of animal semiosis and cognition, including perhaps the realization that mimicry are biological metaphors-biometaphors to live and survive by.

\section{ACKNOWLEDGMENTS}

I would like to thank Anton Markoš, Kalevi Kull, and Timo Maran for their inspiring books and papers in biosemiotics-it was through their work that I first encountered the field of biosemiotics / zoosemiotics. Special thanks belong to the anonymous reviewer(s) who helped with their critical comments. I also owe a great debt of gratitude to many of my students from whom I learn a lot; special thanks belong to Eva Wagenknechtová for her inspiring thirst for knowledge as well as for her practical help with diagrams, visuals and thought-provoking questions. I would also like to thank the young naturalists who shared their animal drawings with me: Jan G. Martinek, Jakob G. Martinek and Eliáš R. Bohát. Last but not least, I would like to express my gratitude to my wife and the whole family for their support and encouragement. SDG.

\section{BIBLIOGRAPHY}

Arenas, L. M., Troscianko, J., Stevens, M., 2014. Color contrast and stability as key elements for effective warning signals. Frontiers in Ecology and Evolution, Vol. 2, 2014. DOI=10.3389/fevo. 2014.00025

Barnhart, Ch. M.; Haag, W. R., Roston, W. N., 2008. Adaptations to host infection and larval parasitism in Unionoida. J. N. Am. Benthol. Soc., 2008, 27(2): 370394 (c) 2008 by The North American Benthological Society DOI: 10.1899/07-093.1

Blount, J. D.; Speed, M. P.; Ruxton, G. D., Stephens, P. A., 2008. Warning displays may function as honest signals of toxicity. Proc. R. Soc. B, 2009, 276, 871877. doi:10.1098/rspb.2008.1407. Published online 18 November 2008

Bostanchi H., Anderson S. C., Kami, H. G., Papenfus, T. J., 2006. "A new species of Pseudocerastes with elaborate tail ornamentation from western Iran (Squamata: Viperidae)," Proc. Calif. Acad. Sci. Ser. 4, 57(14), 443-450

Brown, J. J., Kittelson, N. T., Hannon, E. R., Walsh, D. B., 2006. An endemic population of western poplar clearwing moths (Lepidoptera: Sesiidae) invades a monoculture of hybrid poplar. J. Econ Entomol, 2006 Jun; 99(3): 771-779. doi: 10.1603/0022-049399.3.771. PMID: 16813311.

Campbell, J., 2008. The Hero with a Thousand Faces (The Collected Works of Joseph Campbell) (Third ed.). New World Library.

21 No indisputable evidence seems to be currently known for any human-like moral reasoning or the creation of moral institutions that would penalize "dishonest" signaling by expulsion from the community or any other repercussions. True, absence of evidence in itself is NOT evidence of absence. But in the absence of evidence for analogies of human moral categories in animals it seems more appropriate (and cognitively honest) to follow Wittgenstein's suggestion: "What can be said at all can be said clearly; and whereof one cannot speak thereof one must be silent" (1922, Preface, 23). 
Coletta, W. J., 1993. The Semiotics of Nature: Towards an Ecology of Metaphor and a Biology of Mathematics. The American Journal of Semiotics, 10(3), 223244. https://doi.org/10.5840/ajs1993103/410

Eco, U., 2018. Giorgio Prodi and the lower threshold of semiotics. Sign Systems Studies 46(2/3), 343-351. DOI: 10.12697/SSS.2018.46.2-3.07

Fathinia, B., Rastegar-Pouyani, N., 2010. On the species of Pseudocerastes (Ophidia: Viperidae) in Iran. Russian Journal of Herpetology, Vol. 17, No. 4, 2010, 275-279.

Fathinia, B., Rastegar-Pouyani, N., Rastegar-Pouyani, E., Todehdehghan, F., Amiri, F., 2015. Avian deception using an elaborate caudal lure in Pseudocerastes urarachnoides (Serpentes: Viperidae). Amphib. Reptilia 36, 223-231.

Forbes, P., 2009. Dazzled and Deceived: Mimicry and Camouflage. Yale University Press, 2009.

Frye, N. Jenkins, W. T., 1981. Man the Myth-Maker. Harcourt Brace Jovanovich. New York.

Gohli, J. and Högstedt, Göran. (2010) Reliability in aposematic signaling. Thoughts on evolution and aposematic life. Communicative \& Integrative Biology 3:1, 9-11; January/February 2010; Landes Bioscience.

Haldeman, P., 2016. My Father, the Shapeshifter. The New York Times. December 31, 2016. Available at: <https:// www.nytimes.com/2016/12/31/fashion/susan-faludi-father-in-the-darkroom.html>

Harper, G. R.; Pfennig, D. W., 2007. Mimicry on the edge: Why do mimics vary in resemblance to their model in different parts of their geographical range? Proceedings of the Royal Society B: Biological Sciences 274(1621), 1955-61.

Hyland, A., 2017. Author Susan Faludi on her transgender father, Trump and feminism today. Financial Review. June 2, 2017. https//www.afr.com/life-and-luxury/arts-and-culture/author-susan-faludi-on-her-transfender-father-trump-and-feminism-today-20170523-gwatdm.

Komárek, S., 1998. Mimicry, Aposematism and Related Phenomena (Mimetism in Nature and the History of its Study). LINCOM, Munich.

Komárek, S., 2009. Nature and Culture. The World of Phenomena and the World of Interpretation. LINCOM, Munich.

Kull, K., 2018. Umberto Eco on the biosemiotics of Giorgio Prodi. Sign Systems Studies, 46(2/3), 352-364. https://doi.org/10.12697/SSS.2018.46.2-3.08

Lakoff, G., Johnson, M., 1980. Metaphors we live by. Chicago, IL: University of Chicago Press.

Lakoff, G., 1998. The Contemporary Theory of Metaphor. In Metaphor and Thought (pp. 202-251). Cambridge, UK: Cambridge University Press.

Lebas, N. R., Hockham, L. R., 2005. An invasion of cheats; the evolution of worthless nuptial gifts. Current biology: $C B, 15(1), 64-67$. https://doi.org/10.1016/j. cub.2004.12.043

Lorenz, K., 1973. Analogy as a source of knowledge. Nobel Lecture, December 12, 1973. https://www.nobelprize.org/uploads/2018/06/lorenz-lecture.pdf
Lorenz, K., 2002. King Solomon's Ring. Translated by Marjorie Kerr Wilson. Routledge Classics, London.

Main, D., 2019. Inside the world of treehoppers, mini-marvels of the rainforest. National Geographic Magazine, March 2019. Available at: <https://www.nationalgeographic.com/magazine/2019/03/treehoppers-could-be-worlds-weirdest-insects/?cmpid=org=ngp::mc=crm=-email::src=ngp::.cmp=editorial::addsunstills_20190224:.rid=44075408895>. Accessed: December 31, 2020.

Maran, T., 2007. Semiotic interpretations of biological mimicry. Semiotica 167-1/4 (2007), 223-248. Walter de Gruyter. DOI 10.1515/SEM.2007.077.

Maran, T., 2011. Becoming a sign: The mimic's activity in biological mimicry. Biosemiotics, 4, 243-257.

Maran, T., 2020. Ecosemiotics: The Study of Signs in Changing Ecologies (Elements in Environmental Humanities). Cambridge: Cambridge University Press.

Maran, T., Kull, K., 2014. 'Ecosemiotics: main principles and current developments', Geografiska Annaler: Series B, Human Geography 96 (1): 41-50.

MIPVU (Metaphor Identification Procedure VU University Amsterdam). (n.d.). Retrieved April 22, 2017. Available at: <http://www.vismet.org/metcor/documentation/MIPVU.html>

Nagel, T., 1974. "What Is It Like to Be a Bat?". The Philosophical Review. 83(4), 435-450. doi:10.2307/2183914.

Pilátová, J., 2014. Jak si modrásek povídá s mravenci. Vesmir 93, 675, 2014/12. Prague.

Perel, S., 1997. Europa. John Wiley \& Sons, Inc. New York.

Pragglejaz Group, 2007. MIP: A Method for Identifying Metaphorically Used Words in Discourse. Metaphor and Symbol, 22(1), 1-39. doi:10.1080/10926480709336752

Rosch, E., 1983. Prototype Classification and Logical Classification: The Two Systems. New Trends in Conceptual Representation: Challenges To Piaget's Theory, 73-86. Edited by Ellin Kofsky Scholnick.

Rosch, E., 2013. Principles of Categorization. In Readings in Cognitive Science: A Perspective from Psychology and Artificial Intelligence. UC Berkeley. http://dx.doi.org/10.1016/B978-1-4832-14467.50028-5. Available at: <https://escholarship.org/ uc/item/0sz9c8qh>

Rosch, E., Mervis, C. B., Gray, W. D., Johnson, D. M., Boyes-Braem, P., 1976. Basic objects in natural categories. Cognitive Psychology, Volume 8, Issue 3, 1976, pp. 382-439, ISSN 0010-0285, https://doi. org/10.1016/0010-0285(76)90013-X.

Rota, J., Wagner, D. L., 2006. Predator Mimicry: Metalmark Moths Mimic Their Jumping Spider Predators. PLOS ONE 1(1): e45. doi:10.1371/journal.pone.0000045

Sala M., Casacci L. P., Balletto E., Bonelli S., Barbero F., 2014. Variation in Butterfly Larval Acoustics as a Strategy to Infiltrate and Exploit Host Ant Colony Resources. PLoS ONE 9(4): e94341. https://doi.org/10.1371/ journal.pone.0094341

Schuler, W., Hesse, E., 1985. On the function of warning coloration: a black and yellow pattern inhibits 
prey- attack by naive domestic chicks. Behav Ecol Sociobiol 1985, 249-55.

Sebeok, T., 2001. Signs: An Introduction to Semiotics, second edition (Toronto Studies in Semiotics \& Communication). University of Toronto Press.

Simbeck, R., 2008. For Wildlife Watchers: Eastern coral snake. SC Wildlife Magazine, Jul/Aug 2008. https:// www.dnr.sc.gov/magazine/articles/julyaug2008/coralsnake.html

Speed, M. P., Ruxton, G. D., 2010. Imperfect Batesian Mimicry and the Conspicuousness Costs of Mimetic Resemblance. Am. Nat. 2010. Vol. 176, pp. E1-E14. The University of Chicago. DOI: 10.1086/652990

Stevens, M., Ruxton, G., 2012. Linking the evolution and form of warning coloration in nature. Proc Biol Sci. 2012 Feb 7; 279(1728): 417-426. doi: 10.1098/ rspb.2011.1932

Summers, K., Speed, M. P., Blount, J. D., Stuckert, A. M. M., 2015. Are aposematic signals honest? A review. J. EVOL. BIOL. 28 (2015) 1583-1599. European Society for Evolutionary Biology.

Thomas, J. A., Wardlaw, J. C., 1990. The effect of queen ants on the survival of Maculinea arion larvae in Myrmica ant nests. Oecologia 85, 87-91. https://doi. org/10.1007/BF00317347

Thomas, J. A.,Elmes, G. W., 1998. Higher productivity at the cost of increased host-specificity when Maculinea butterfly larvae exploit ant colonies through trophallaxis rather than by predation.
Ecological Entomology 23, 457-464. https://doi. org/10.1046/j.1365-2311.1998.00153.x

Thomas, J. A., Elmes, G. W., Sielezniew M., Stankiewicz-Fiedurek, A., Simcox D. J., Settele J., Schönrogge, K., 2013. Mimetic host shifts in an endangered social parasite of ants. Proc. R. Soc. B. 28020122336 http://doi.org/10.1098/rspb.2012.2336

Tseng, L., Tso, I. M., 2009. A risky defence by a spider using conspicuous decoys resembling itself in appearance. Animal Behaviour, 78, 425-431. https://doi. org/10.1016/j.anbeh av.2009.05.017

Walker, G. P., 2009. Chapter 228-Salivary Glands. In: Resh, V., Cardé, R. T. (Eds.), Encyclopedia of Insects (Second Edition). Academic Press, pp. 897-901, https://doi. org/10.1016/B978-0-12-374144-8.00237-X.

Williams, L., 2011. Nature mimics: why bugs mate with beer bottles. Australian Geographic, November 30, 2011. Available at: <https://www.australiangeographic.com.au/topics/wildlife/2011/11/ nature-mimics-why-bugs-mate-with-beer-bottles/>

Wittgenstein, L., 1922. Tractatus Logico-Philosophicus. Kegan Paul, Trench, Trubner \& Co., Ltd. London.

Return to front page $\uparrow$ 